\title{
Beyond finding druggable targets and tumor mutational burden: to measure heterogeneity by ctDNA
}

\author{
Zhichao Liu ${ }^{1,2}$, Jianxing $\mathrm{He}^{1}$, Wenhua Liang ${ }^{1}$ \\ ${ }^{1}$ Department of Thoracic Surgery and Oncology, the First Affiliated Hospital of Guangzhou Medical University, State Key Laboratory of Respiratory \\ Disease, National Clinical Research Center for Respiratory Disease, Guangzhou Institute of Respiratory Health, Guangzhou, China; ${ }^{2}$ Department of \\ Thoracic Surgery, Shanghai Chest Hospital, Shanghai Jiao Tong University, Shanghai, China \\ Correspondence to: Wenhua Liang. Department of Thoracic Surgery and Oncology, the First Affiliated Hospital of Guangzhou Medical University, \\ State Key Laboratory of Respiratory Disease, National Clinical Research Center for Respiratory Disease, Guangzhou Institute of Respiratory \\ Health, No. 151, Yanjiang Rd, Guangzhou, China. Email: liangwh1987@163.com. \\ Provenance and Peer Review: This article was commissioned by the editorial office, Annals of Translational Medicine. The article did not undergo \\ external peer review. \\ Comment on: Liu Z, Xie Z, Zhao S, et al. Presence of allele frequency heterogeneity defined by ctDNA profiling predicts unfavorable overall survival \\ of NSCLC. Transl Lung Cancer Res 2019;8:1045-50.
}

Submitted Jan 14, 2020. Accepted for publication Jan 30, 2020.

doi: $10.21037 /$ atm.2020.01.86

View this article at: http://dx.doi.org/10.21037/atm.2020.01.86

With the advances in molecular oncology, genomic analysis for selection of molecularly guided therapy has become standard of care for various advanced solid tumors $(1,2)$. Tumor sequencing not only helps to inform which druggable targets are likely to be effective [e.g., EGFR-TKI in EGFR-mutant non-small cell lung cancer (NSCLC)] but also provides many promising biomarkers in guiding cancer immunotherapy [e.g., tumor mutational burden (TMB) and microsatellite instability (MSI)]. The genomic sequencing continues to evolve, and our understanding of the landscape of tumor heterogeneity must advance accordingly to continue to improve outcomes for patients. Circulating tumor DNA (ctDNA), a noninvasive tool to detect genetic mutations in cancer patients, has been increasingly accessible worldwide and provides a comprehensive landscape across cancerous tissue for making it an ideal approach to assess the tumor heterogeneity (3-5). In this issue of Translational Lung Cancer Research, we investigated the value of heterogeneity of allele frequency (AF) defined by ctDNA mutations in the prognosis of advanced NSCLC in a real-world data analysis (6), which tried to provide a new insight into tumor heterogeneity from the ctDNA level. This study highlights the importance of further molecular analysis at the current ctDNA profiling as novel potentially biomarkers associated with tumor biology may thus be discovered.
Cancer is a disease of complex genetic mutations (7), and comprehensive genomic analysis is beneficial to help match patient to appropriate treatment. Although genomic analysis is generally done in the current clinical setting, a lack of tumor heterogeneity information in the routine genomic analysis could be a barrier and challenge for appropriate treatment decisions. Tumor genomic heterogeneity is presumed to be a fundamental cause of therapeutic failure $(8,9)$. However, researchers are still at the very beginning of understanding the full extent of tumor heterogeneity. Challenge in assessing tumor heterogeneity lies in the fact that currently there exists no systematic understanding to it and the evaluation tools are lacking relatively mature for studying tumor mutation evolution and quantifying heterogeneity.

Ongoing efforts to integrally analysis the tumor mutation data may help incorporate relevant features that associated with tumor heterogeneity. Despite the routine genetic test provides information about genetic variants, druggable targets and TMB, it does not cover the information of tumor heterogeneity. ctDNA is shed into the bloodstream from tumors, it is thereby a genomic reservoir of different tumor clones and a good representation of tumor genomic diversity (10). And theoretically, ctDNA may attenuate the issues related to assessing tumor heterogeneity, which provide a possible way to dig out 
the tumor heterogeneity information that hidden behind tumor mutations. Therefore, as described above, our team made an investigation to explore the effect of $\mathrm{AF}$ of ctDNA mutations in the prognosis of advanced NSCLC.

The AF variant of different genetic mutations are often used to represent the clonal makeup of a tumor, and lower $\mathrm{AF}$ mutations are more likely to be subclonal $(5,11)$, which potentially represent tumor heterogeneity. Therefore, our study hypothesized that relatively low frequency mutations in ctDNA might indicate tumor heterogeneity, or more specifically, allele frequency heterogeneity (AFH). A low ratio of $\mathrm{AF}$ of a mutation to the maximum somatic allele frequency (MSAF) was used to define the presence of AFH. By using trend regression analysis, the low ratio of $\mathrm{AF} /$ MSAF and overall survival were correlated and the cutpoint of AF/MSAF $<10 \%$ was founded and confirmed; the presence of mutations with low ratio of AF/MSAF $<10 \%$ was thus applied for defining the presence of $\mathrm{AFH}$. The result indicated that the presence of $\mathrm{AFH}$ (case with mutations of AF/MSAF < $10 \%$ ) may provide a poorer prognosis among patients with advanced NSCLC.

Different types of patients may present diverse treatment strategy and survival outcome, therefore, we examined the difference of treatment types, trying to find out more details of the impact of AFH on overall survival. The results demonstrated that the presence of AFH (cases with mutation of AF/MSAF < $10 \%$ ) was an adverse prognostic factor for overall survival across treatment arms (chemotherapy, immunotherapy for patients without actionable driver mutation; and EGFR-TKIs for patients with EGFR mutation), which gave important assumption that the AFH defined by AF/MSAF $<10 \%$ might be a universal prognostic factor across different subsets of advanced NSCLC. This might emphasize the importance of AFH evaluation for all advanced NSCLC patients.

One of the merits of this study is to determine that ctDNA mutation with low ratio of AF/MSAF $<10 \%$ presents the poorer overall survival in advanced NSCLC patients. Since the evaluation of tumor heterogeneity has not yet arrived in the current clinical practice, it is hard to obtain comprehensive information of tumor heterogeneity; however, ctDNA profiling has been widely accessible nowadays. Therefore, maximizing full value of ctDNA profiling results as much as possible may be considered as a feasible complementary strategy. This study initially links relatively low frequency mutations (AFH) in ctDNA profiling to prognostic outcome by using accessible indicators (AF/MSAF $<10 \%)$. By adding the simple calculation to the current ctDNA genomic test report, without further cost, the tumor heterogeneity might be indicated by $\mathrm{AFH}$ evaluation and adjusting a therapeutic regimen may be accordingly.

Advanced analysis of the current genomic test results integrated with clinical characteristics, will lead to further understanding of tumor biology and improve patient outcomes. In our investigation, we demonstrated the significance of relatively low-frequency mutations defined by ctDNA profiling in the prognosis of advanced NSCLC and provided a novel prognostic biomarker, namely AFH, a genetic heterogeneity feature. This study contributes to shed the light on the effects of $\mathrm{AFH}$ in the prognosis of advanced NSCLC. Clinicians should pay more attention to the clinical significance of genomic test results in therapy strategy.

In conclusion, presence of $\mathrm{AFH}$ is an independent predictive factor for poor prognosis in advanced NSCLC. Besides the conventional druggable targets and TMB, more information of tumor biology could be provided through further ctDNA profiling. By adding this easily calculated AFH to the current ctDNA profiling, which incurs no further cost, we can additionally evaluate or monitor tumor heterogeneity and its prognosis. Further research has to focus on this issue through more comprehensive analysis and experimental evaluation to confirm the association between $\mathrm{AFH}$ and prognosis.

\section{Acknowledgments}

Funding: None.

\section{Footnote}

Conflicts of Interest: All authors have completed the ICMJE uniform disclosure form (available at http://dx.doi. org/10.21037/atm.2020.01.86). JH serves as an unpaid editorial board member of Annals of Translational Medicine from Jun 2019 to May 2024. The other authors have no conflicts of interest to declare.

Ethical Statement: The authors are accountable for all aspects of the work in ensuring that questions related to the accuracy or integrity of any part of the work are appropriately investigated and resolved.

Open Access Statement: This is an Open Access article distributed in accordance with the Creative Commons 
Attribution-NonCommercial-NoDerivs 4.0 International License (CC BY-NC-ND 4.0), which permits the noncommercial replication and distribution of the article with the strict proviso that no changes or edits are made and the original work is properly cited (including links to both the formal publication through the relevant DOI and the license). See: https://creativecommons.org/licenses/by-nc-nd/4.0/.

\section{References}

1. Senft D, Leiserson MDM, Ruppin E, et al. Precision Oncology: The Road Ahead. Trends Mol Med 2017;23:874-98.

2. Oxnard GR, West HJ, King JC. Improving Molecular Oncology by Making Results Available to Patients. JAMA Oncol 2019. [Epub ahead of print].

3. Murtaza M, Dawson SJ, Tsui DW, et al. Non-invasive analysis of acquired resistance to cancer therapy by sequencing of plasma DNA. Nature 2013;497:108-12.

4. Wang Z, Cheng Y, An T, et al. Detection of EGFR mutations in plasma circulating tumour DNA as a selection criterion for first-line gefitinib treatment in patients with advanced lung adenocarcinoma (BENEFIT): a phase 2,

Cite this article as: Liu Z, He J, Liang W. Beyond finding druggable targets and tumor mutational burden: to measure heterogeneity by ctDNA. Ann Transl Med 2020;8(11):723. doi: 10.21037/atm.2020.01.86 single-arm, multicentre clinical trial. Lancet Respir Med 2018;6:681-90.

5. Williams MJ, Werner B, Barnes CP, et al. Identification of neutral tumor evolution across cancer types. Nat Genet 2016;48:238-44.

6. Liu Z, Xie Z, Zhao S, et al. Presence of allele frequency heterogeneity defined by ctDNA profiling predicts unfavorable overall survival of NSCLC. Transl Lung Cancer Res 2019;8:1045-50.

7. Meacham CE, Morrison SJ. Tumour heterogeneity and cancer cell plasticity. Nature 2013;501:328-37.

8. Dagogo-Jack I, Shaw AT. Tumour heterogeneity and resistance to cancer therapies. Nat Rev Clin Oncol 2018;15:81-94.

9. Kleppe M, Levine RL. Tumor heterogeneity confounds and illuminates: assessing the implications. Nat Med 2014;20:342-4.

10. Diaz LA Jr, Bardelli A. Liquid biopsies: genotyping circulating tumor DNA. J Clin Oncol 2014;32:579-86.

11. Chae YK, Davis AA, Agte $S$, et al. Clinical Implications of Circulating Tumor DNA Tumor Mutational Burden (ctDNA TMB) in Non-Small Cell Lung Cancer. Oncologist 2019;24:820-8. 